\title{
Le budget global s'invite par la petite porte
}

\section{Jürg Schlup}

Dr méd., président de la FMH

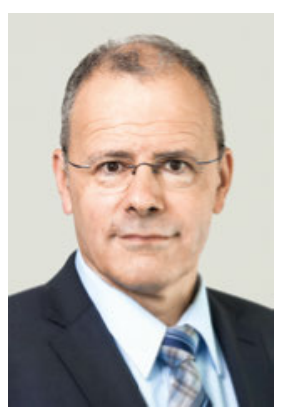

Jusqu'ici, le rapport d'experts sur les mesures visant à freiner les coûts [1], réalisé à la demande du Département fédéral de l'intérieur (DFI), a surtout été débattu parce qu'il propose d'instaurer un plafonnement collectif des coûts pour les traitements des patients. Malgré le danger que cette mesure représente pour notre système de santé, il ne faut pas perdre de vue les 37 autres propositions du rapport. En février 2018, la FMH a présenté ses propres propositions, qui recoupent en partie celles des experts [2]. Les prochaines étapes de la mise en œuvre des nombreuses mesures prévues dans le rapport restent encore à définir.

\section{En tant que médecins, nous sommes tenus} de mettre en garde contre les pseudo-remèdes promettant une efficacité maximale sans aucun effet secondaire.

Le 28 mars 2018, le Conseil fédéral a donné la priorité à neuf des 38 mesures du rapport ainsi qu'à trois propositions supplémentaires du DFI [3]. La FMH a pris position sur ces différentes mesures (voir p. 604 et suivantes) [4]: nous sommes particulièrement favorables à l'idée de réduire les conflits de gouvernance des cantons et nous approuvons l'amélioration du contrôle des factures. De même, l'article expérimental, jugé prioritaire par le Conseil fédéral, pourrait à notre point de vue contribuer au développement du système de santé, pour autant que les fournisseurs de prestations soient associés aux projets pilotes et que les indicateurs utilisés pour évaluer ces derniers soient définis de manière conjointe et transparente. Or malheureusement, les fournisseurs de prestations sont mis à l'écart et les cinq mesures portant sur le tarif ambulatoire nous restreignent toujours davantage, alors que la Confédération souhaite étendre ses compétences, notamment en tant qu'«autorité d'adaptation et de fixation" ([1], p. 80). Parce que la FMH connaît mieux que quiconque les travaux en cours dans le domaine des tarifs, nous saluons tout changement susceptible d'apporter des améliorations. Mais de plus en plus de compétences étatiques - même subsidiaires - sont contre-productives parce qu'elles nuisent au partenariat tarifaire. Aujourd'hui, elles vident déjà l'autonomie tarifaire de son sens en rendant un échec des négociations trop attractif pour certains partenaires. Si l'on ne souhaite pas ouvrir délibérément la voie à un tarif d'Etat, les réformes imaginées doivent être placées sous le signe de l'autonomie structurelle et organisationnelle des partenaires tarifaires.

Dans ce contexte, le "pilotage des coûts par les partenaires tarifaires», tel qu'il est proposé par le DFI et qui compte parmi les priorités du Conseil fédéral, s'avère particulièrement problématique [3]. L'obligation légale faite aux fournisseurs de prestations et aux assureurs «de prévoir des mesures de pilotage des coûts et des prestations dans leurs conventions tarifaires» [3] afin de freiner la hausse des coûts - mais encore une fois avec la compétence subsidiaire de la Confédération ou des cantons - est une tentative pour faire entrer le budget global par la petite porte: lorsqu'à l'avenir les politiques jugeront les prestations médicales trop coûteuses, les partenaires tarifaires seront accusés de mal piloter les coûts - et l'Etat prendra le relais. Le "pilotage» (en réalité, le rationnement) de la prise en charge des patients selon des critères politiques est une tâche que nous refusons d'assumer. Nous garderons donc cette porte dérobée fermée!

Le rationnement de la prise en charge des patients est une tâche que nous refusons d'assumer.

Il n'est toutefois pas certain que cela suffise, si PDC et Confédération sont prêts à défoncer la porte principale à coups de bélier. Promettre à l'électorat une maîtrise des coûts sans coupe douloureuse, tout en améliorant la qualité, semble être aujourd'hui une promesse politique à succès. En tant que médecins, nous sommes tenus de mettre en garde contre les pseudo-remèdes promettant une efficacité maximale sans aucun effet secondaire. Bien que ce soit assurément une tâche impopulaire, nous l'accomplirons sans faillir. 